\title{
What drives fluctuations of labor wedge and business cycles? Evidence from Japan
}

Masaru Inaba (Kansai University / The Canon Institute for Global Studies)

Kengo Nutahara (Senshu University / The Canon Institute for Global Studies)

Daichi Shirai (Tohoku Gakuin University/ The Canon Institute for Global Studies)

2020.08

※Opinions expressed or implied in the CIGS Working Paper Series are solely those of the author, and do not necessarily represent the views of the CIGS or its sponsor.

※CIGS Working Paper Series is circulated in order to stimulate lively discussion and comments. ※Copyright belongs to the author(s) of each paper unless stated otherwise.

General Incorporated Foundation

The Canon Institute for Global Studies

一般財団法人 キヤノングローバル戦略研究所

Phone: +81-3-6213-0550 http://www.canon-igs.org 


\title{
What drives fluctuations of labor wedge and business cycles? Evidence from Japan *
}

\author{
Masaru Inaba \\ Kengo Nutahara ${ }^{\dagger}$ \\ Kansai University and CIGS Senshu University and CIGS \\ Daichi Shirai \\ Tohoku Gakuin University and CIGS
}

August 14, 2020

*This work is financially supported by the Zengin Foundation for Studies on Economics and Finance in 2018 and the Research Institute for Socionetwork Strategies of Kansai University in 2020.

${ }^{\dagger}$ Corresponding Author; Address: 2-1-1 Higashimita, Tama-ku, Kawasaki, Kanagawa 214-8580, Japan. E-mail: nutti@isc.senshu-u.ac.jp, Tel: 044-911-1230, Fax: 044-911-1231. 


\begin{abstract}
It is well known that the labor wedge worsens in the recessions. The main research of this study are as follows. (i) What is the main driving force of the labor wedge? (ii) Is the main driver of the labor wedge the same as that of business cycles? In this study, we analyze which structural shocks drive the fluctuation of the labor wedge and business cycles using a canonical medium-scale dynamic stochastic general equilibrium model with nominal and real frictions. The model is estimated using Japanese data. One of the novel features of this study is our estimation strategy. In standard Bayesian estimation, the prior distribution of the parameters for the standard deviations of the structural shocks is inverse gamma, which does not support zero value and assumes the existence of structural shocks. On the contrary, we employ a more flexible prior distribution of the parameters for the standard deviations of structural shocks to allow the non-existence of structural shocks. Under the standard prior distribution, the estimation results imply that the labor wedge is mainly driven by preference and transitory technology shocks, whereas the investment adjustment cost shock is the most important for the business cycle fluctuations. However, under our relaxed prior distribution, which allows the non-existence of structural shocks, the estimation results imply that both the labor wedge and the business cycles are mainly driven by permanent technology and investment adjustment cost shocks.
\end{abstract}

Keywords: Labor wedge; DSGE model; structural shocks; measurement error; prior distribution

JEL codes: E32; E37 


\section{Introduction}

It is well known that the labor wedge, defined as the gap between the marginal rate of substitution and the marginal productivity of labor, worsens in recessions, including the Great Depression and Great Recession. The labor wedge has been investigated as an important variable to understand business cycle fluctuations.

The main research questions of this study are as follows. (i) What is the main driving force of the labor wedge in a canonical medium-scale dynamic stochastic general equilibrium (DSGE) economy? (ii) Is the main driver of the labor wedge the same as that of business cycles? The recent literature on the medium-scale DSGE model has found that such a model can account for the salient aspects of business cycle fluctuations. In the literature, many works investigate the main driving force of output fluctuations, whereas there are few studies on the source of the fluctuations of the labor wedge. ${ }^{1}$ If the labor wedge is an important variable to understand business cycles, then the main driving force of the labor wedge should be the same as that of business cycles.

In this study, we analyze which structural shocks drive the fluctuation of the labor wedge and business cycles using a canonical medium-scale DSGE model. There are many nominal and real frictions, and many structural shocks in the model. We estimate the model using Japanese data. One of special features of this study is our estimation strategy. In the standard Bayesian estimation of DSGE models, the prior distribution of the parameters for the standard deviations of the shocks is the inverse gamma distribution, which does not support zero value and assumes the existence of structural shocks. On the contrary, in this study, we employ a more flexible prior distribution of the parameters for the standard deviations of shocks and measurement errors to allow the non-existence of shocks and measurement errors. Following Ferroni, Grassi, and

\footnotetext{
${ }^{1}$ An exception is Cheremukhin and Restrepo-Echavarria (2014), who investigate the source of the labor wedge in a DSGE model with search and matching in the labor market, while our model is a canonical medium-scale DSGE model without search and matching frictions.
} 
Leon-Ledesma (2019), we employ normal distribution in this study.

Under standard prior distribution of the parameters for the standard deviations of structural shocks, the estimation results imply that the labor wedge is mainly driven by preference and transitory technology shocks, whereas the investment adjustment cost shock is the most important for business cycle fluctuations. Meanwhile, under our relaxed prior distribution, which allows the non-existence of shocks, the estimation results show that both the labor wedge and the business cycles are mainly driven by permanent technology and investment adjustment cost shocks.

Our estimation results imply the followings. If our estimation were to employ inverse gamma distribution, which is standard in the literature but assumes the existence of structural shocks, then the investigation of the source of the labor wedge fluctuation might not be promising to understand business cycles. This is because the source of the labor wedge is different from that of business cycles. However, by using our more relaxed prior, the normal distribution, to allow the non-existence of structural shocks, the investigation of the labor wedge would be promising to understand business cycles: both the labor wedge and business cycles are driven by the same structural shocks.

Related literature: The importance of the labor wedge in business cycles is emphasized by Chari, Kehoe, and McGrattan (2002, 2007), Kobayashi and Inaba (2006), Shimer (2009), Ohanian (2010), and Otsu (2011). Chari, Kehoe, and McGrattan (2002, 2007) propose the business cycle accounting method and find that the labor wedge is important for the US Great Depression and the 1982 recession. Kobayashi and Inaba (2006) find that the labor wedge is helpful to account for the Japan's lost decade during the 1990s and the recession during the 1920s. Shimer (2009) and Ohanian (2010) find that the labor wedge worsens during recession. In particular, Ohanian (2010) focuses on the US Great Recession. Otsu (2011) investigates the dynamics of the labor wedge of Japanese economy from 1980 to 2007. 
The main driving force of the fluctuation of the labor wedge is investigated by Hall (2009), Shimer (2009), Pescatori and Tasci (2011), Cheremukhin and RestrepoEchavarria (2014), Karabarbounis (2014a, 2014b), Duras (2017), and Zhang (2018). Hall (2009), Shimer (2009), Pescatori and Tasci (2011), and Cheremukhin and RestrepoEchavarria (2014) emphasize the role of matching frictions in the labor market. Duras (2017) finds that matching frictions in the goods market, in addition to the labor market, help to account for fluctuations of the labor wedge.

Karabarbounis (2014a) empirically finds that fluctuations of the labor wedge mainly reflect fluctuations of the gap between the real wage and the marginal rate of substitution. He emphasizes the importance of modeling the household side of the labor market. Karabarbounis (2014b) proposes that home production in the utility function accounts for the international findings of the labor wedge. Zhang (2018) finds that the collateral constraints of entrepreneurs is helpful to account for the variation in the labor wedge during the credit crunch.

Our medium-scale DSGE model does not include matching frictions, home production, and collateral constraints, as investigated by these existing works. In our model, habit persistence and sticky wage are considered, generating a gap between the marginal rate of substitution and the real wage. This setup is consistent with the empirical finding of Karabarbounis (2014a).

The sources of business cycle fluctuations have been investigated by many researchers. In particular, the importance of technology shock is emphasized traditionally as in King and Rebelo (1999) and Hayashi and Prescott (2002). Recent works by Kaihatsu and Kurozumi (2014a, 2014b) find that technology shocks are the main driving force of business cycles both in the US and Japan. Meanwhile, Justiniano, Primiceri, and Tambalotti (2010, 2011) and Hirose and Kurozumi (2012) emphasize the importance of the investment adjustment cost shock in business cycles. In our estimate, the (permanent) technology and investment adjustment cost shocks are important for business cycles, 
which are consistent with these findings in the previous research.

Our estimation method is based on Ferroni, Grassi, and Leon-Ledesma (2019), who propose an estimation method allowing the non-existence of structural shocks. The authors find that government spending, price markup, and wage markup shocks do not generate significant dynamics using the model of Smets and Wouters (2007) in the US.

The remainder of the paper is organized as follows. Section 2 introduces the model. Section 3 shows the data and the estimation strategy of the model. Section 4 explains the main results. Finally, Section 5 concludes.

\section{The Model}

\subsection{The Model}

The model is a variant of the medium-scale DSGE model à la Christiano, Eichenbaum, and Evans (2005) and Smets and Wouters (2007). There are many real and nominal frictions to account for the salient aspects of business cycles. As real frictions, habit persistence, adjustment costs of investment, and variable capital utilization are introduced. As nominal frictions, Calvo-type nominal price and wage rigidities with partial inflation indexations are introduced. The central bank follows a Taylor-type nominal interest rate rule. There are many structural shocks: permanent and transitory technology, preference (a shock to the discount factor), labor supply (a shock to the weight of disutility from labor supply), investment adjustment cost, price markup, wage markup, government purchases, and monetary policy shocks. ${ }^{23}$

\footnotetext{
${ }^{2}$ In our model, the investment specific technology shock is eliminated from the model of Hirose and Kurozumi (2012), and the transitory technology shock is introduced.

${ }^{3}$ As explained in section 2.2, we redefine the wage shock as the linear combination of the labor supply and wage markup shocks, because the labor supply and wage markup shocks cannot be identified in this model.
} 
Final-good firms: The final-good firms are perfectly competitive. They produce homogeneous final-good $Y_{t}$ using intermediate-good $Y_{t}(f)$. The production function is given by

$$
Y_{t}=\left[\int_{0}^{1} Y_{t}(f)^{\frac{1}{1+\lambda_{t}^{p}}} d f\right]^{1+\lambda_{t}^{p}},
$$

where $\lambda_{t}^{p}$ is a time-varying parameter for elasticity of substitution among intermediate$\operatorname{good} \theta_{t}^{p}>1$, which is defined by $\lambda_{t}^{p}=1 /\left(\theta_{t}^{p}-1\right)>0$. Profit maximization implies the demand function of intermediate-good $Y_{t}(f)$ :

$$
Y_{t}(f)=\left[\frac{P_{t}(f)}{P_{t}}\right]^{-\frac{1+\lambda_{t}^{p}}{\lambda_{t}^{p}}} Y_{t},
$$

where $P_{t}$ is the price of final-good $Y_{t}$, and $P_{t}(f)$ is the price of intermediate-good $Y_{t}(f)$.

Intermediate-good firms: The intermediate-good firms are monopolistically competitive. The intermediate-good firm indexed by $f \in[0,1]$ produces differentiated intermediate-good $Y_{t}(f)$ using labor input $\ell_{t}(f)$ and capital service $K_{t}^{S}(f)$. The production function is given by

$$
Y_{t}(f)=\exp \left(z_{t}^{z t}\right)\left[K_{t}^{S}(f)\right]^{\alpha}\left[Z_{t}^{P} \ell_{t}(f)\right]^{1-\alpha}-\Phi Z_{t}^{P},
$$

where $\alpha \in(0,1)$ is the cost share of capital; $\Phi$ is the fixed cost of production; $z_{t}^{z t}$ is the transitory technology shock; and $Z_{t}^{P}$ is the permanent technology that evolves according to

$$
\log Z_{t}^{p}=z^{*}+\log Z_{t-1}^{P}+z_{t}^{z p}
$$

where $z^{*}$ is the steady-state growth rate of $Z_{t}^{P}$, and $z_{t}^{z p}$ is the permanent technology shock. Both $z_{t}^{z t}$ and $z_{t}^{z p}$ follow the $\mathrm{AR}(1)$ process. The last term in the production function, $\Phi Z_{t}^{P}$, is multiplied by $Z_{t}^{P}$ to guarantee the existence of the balanced growth path. The cost 
minimization of intermediate-good firms implies

$$
\begin{aligned}
& R_{t}^{k}=m c_{t} \alpha \exp \left(z_{t}^{z t}\right)\left[\frac{Z_{t}^{P} \ell_{t}(f)}{K_{t}^{S}(f)}\right]^{1-\alpha}, \\
& W_{t}=m c_{t}(1-\alpha) \exp \left(z_{t}^{z t}\right) Z_{t}^{P}\left[\frac{Z_{t}^{P} \ell_{t}(f)}{K_{t}^{S}(f)}\right]^{-\alpha},
\end{aligned}
$$

where $m c_{t}$ is the real marginal cost; $R_{t}^{k}$ is the rental rate of capital; and $W_{t}$ is the real wage rate.

Calvo-type sticky prices are introduced. In every period, a fraction $\xi_{p} \in[0,1]$ of intermediate-good firms can reoptimize their prices. The other firms index their prices to the weighted average of past inflation $\left(\pi_{t-1}\right)$ and the steady-state inflation $\left(\pi^{*}\right)$ : $\pi_{t-1}^{\gamma_{p}}\left(\pi^{*}\right)^{1-\gamma_{p}}$, where $\gamma_{p} \in[0,1]$ is the relative weight of the past inflation. The objective function of the intermediate-good firms that reoptimize their prices at period $t$ is

$$
E_{t} \sum_{j=0}^{\infty}\left(\beta \xi_{p}\right)^{j}\left(\frac{\Lambda_{t+j}}{\Lambda_{t}}\right)\left[\frac{P_{t}(f)}{P_{t+j}} \prod_{k=1}^{j}\left(\pi_{t+k-1}^{\gamma_{p}}\left(\pi^{*}\right)^{1-\gamma_{p}}\right)-m c_{t+j}\right] Y_{t+j}(j)
$$

where $\Lambda_{t}$ is the marginal utility of consumption of households, and $\beta^{j} \frac{\Lambda_{t+j}}{\Lambda_{t}}$ is the stochastic discount factor. The demand function for $Y_{t+j}(f)$ is given by

$$
Y_{t+j}(f)=\left[\frac{P_{t}(f)}{P_{t+j}} \prod_{k=1}^{j}\left(\pi_{t+k-1}^{\gamma_{p}}\left(\pi^{*}\right)^{1-\gamma_{p}}\right)\right]^{-\frac{1+\lambda_{t+j}^{p}}{\lambda_{t+j}^{p}}} Y_{t+j} .
$$

The reoptimized price $P_{t}^{o}$ is the same for all intermediate-good firms. The first-order condition for reoptimized price $P_{t}^{o}$ is

$$
1=\frac{E_{t} \sum_{j=0}^{\infty}\left(\beta \xi_{p}\right)^{j} \frac{\left(1+\lambda_{t+j}^{p}\right) m c_{t+j} \Lambda_{t+j} Y_{t+j}}{\lambda_{t+j}^{p}}\left[\frac{P_{t}^{0}}{P_{t}} \prod_{k=1}^{j}\left(\frac{\pi_{t+k-1}}{\pi^{*}}\right)^{\gamma_{p}} \frac{\pi^{*}}{\pi_{t+k}}\right]^{-\frac{1+\lambda_{t+j}^{p}}{\lambda_{t+j}^{p}}}}{E_{t} \sum_{j=0}^{\infty}\left(\beta \xi_{p}\right)^{j} \frac{\Lambda_{t+j} Y_{t+j}}{\lambda_{t+j}^{p}}\left[\frac{P_{t}^{0}}{P_{t}} \prod_{k=1}^{j}\left(\frac{\pi_{t+k-1}}{\pi^{*}}\right)^{\gamma_{p}} \frac{\pi^{*}}{\pi_{t+k}}\right]^{-\frac{1}{\lambda_{t+j}^{p}}}} .
$$

Households: The household indexed by $h \in[0,1]$ consumes $C_{t}(h)$, invests $I_{t}(h)$, holds safe asset $B_{t}(h)$ and capital stock $K_{t}(h)$, and supplies differentiated labor service $\ell_{t}(h)$. 
The utility function is given by

$$
E_{0} \sum_{t=0}^{\infty} \beta^{t} \exp \left(z_{t}^{b}\right)\left[\frac{\left[C_{t}(h)-\theta C_{t-1}(h)\right]^{1-\sigma}}{1-\sigma}-\frac{Z_{t}^{1-\sigma} \exp \left(z_{t}^{\ell}\right) \ell_{t}(h)^{1+\chi}}{1+\chi}\right],
$$

where $\beta \in(0,1)$ denotes the discount factor; $\sigma>0$ is the elasticity of intertemporal substitution; $\theta \in(0,1)$ is the degree of habit persistence; $\chi>0$ denotes the inverse of the labor supply elasticity; and $z_{t}^{b}$ and $z_{t}^{\ell}$ are the structural shocks to discount factor (preference shock) and labor supply (labor supply shock), respectively. The technology term $Z_{t}^{1-\sigma}$ appears in the disutility of labor supply, as employed by Erceg, Guerrieri, and Gust (2006), to ensure the existence of a balanced growth path.

The budget constraint of the household is

$$
C_{t}(h)+I_{t}(h)+\frac{B_{t}(h)}{P_{t}}=W_{t}(h) \ell_{t}(h)+R_{t}^{k} u_{t}(h) K_{t-1}(h)+\frac{R_{t-1}^{n} B_{t-1}(h)}{P_{t}}+T_{t}(h),
$$

where $P_{t}$ denotes the price level; $W_{t}(h)$ is the real wage rate; $R_{t}^{n}$ is the nominal gross interest rate; $R_{t}^{k}$ denotes the real rental rate of capital; $u_{t}(h)$ is the capital utilization rate; $K_{t-1}(h)$ is capital stock at the end of period $t-1$; and $T_{t}(h)$ denotes a transfer from the government and firms.

The capital stock evolves as follows:

$$
K_{t}(h)=\left[1-\delta\left(u_{t}(h)\right)\right] K_{t-1}(h)+\left[1-S\left(\frac{I_{t}(h)}{I_{t-1}(h)} \frac{\exp \left(z_{t}^{i}\right)}{z^{*}}\right)\right] I_{t}(h),
$$

where $z_{t}^{i}$ is the investment adjustment cost shock. In this specification, the cost of high capital utilization is a high depreciation rate of capital as in Greenwood, Hercowitz, and Huffman (1988); $\delta^{\prime}(\cdot)>0, \delta^{\prime \prime}(\cdot)>0$, and $\delta^{\prime}\left(u^{*}\right) / \delta^{\prime \prime}\left(u^{*}\right)=\mu$, where $u^{*}$ is the steady-state capital utilization rate. The functional form of the adjustment costs of investment is given by

$$
S(x)=\frac{1}{\zeta}(x-1)^{2}
$$

where $1 / \zeta>0$ is the degree of adjustment cost of investment. 
Because of the existence of complete insurance markets, the decisions of $C_{t}(h), B_{t}(h)$, $u_{t}(h), K_{t}(h)$, and $I_{t}(h)$ are the same for all households; then, the first-order conditions are given by

$$
\begin{aligned}
& \Lambda_{t}=\exp \left(z_{t}^{b}\right)\left[C_{t}-\theta C_{t-1}\right]^{-\sigma}-\beta \theta E_{t}\left(\exp \left(z_{t+1}^{b}\right)\left[C_{t+1}-\theta C_{t}\right]\right), \\
& \Lambda_{t}=\beta E_{t}\left[\Lambda_{t+1} \cdot \frac{R_{t}^{n}}{\pi_{t+1}}\right], \\
& \begin{aligned}
& R_{t}^{k}=Q_{t} \delta^{\prime}\left(u_{t}\right), \\
& 1=Q_{t} {\left[1-S\left(\frac{I_{t}}{I_{t-1}} \frac{\exp \left(z_{t}^{i}\right)}{z^{*}}\right)-S^{\prime}\left(\frac{I_{t}}{I_{t-1}} \frac{\exp \left(z_{t}^{i}\right)}{z^{*}}\right) \frac{I_{t}}{I_{t-1}} \frac{\exp \left(z_{t}^{i}\right)}{z^{*}}\right] } \\
& \quad+\beta E_{t}\left[\frac{\Lambda_{t+1}}{\Lambda_{t}} Q_{t+1} S^{\prime}\left(\frac{I_{t+1}}{I_{t}} \frac{\exp \left(z_{t+1}^{i}\right)}{z^{*}}\right)\left(\frac{I_{t+1}}{I_{t}}\right)^{2} \frac{\exp \left(z_{t+1}^{i}\right)}{z^{*}}\right], \\
& Q_{t}=\beta E_{t} {\left[\frac{\Lambda_{t+1}}{\Lambda_{t}}\left\{R_{t+1}^{k} u_{t+1}+Q_{t+1}\left(1-\delta\left(u_{t+1}\right)\right)\right\}\right], }
\end{aligned}
\end{aligned}
$$

where $\Lambda_{t}$ is the marginal utility of consumption; $\pi_{t+1}=P_{t+1} / P_{t}$ is the gross price inflation rate; and $Q_{t}$ is the real price of capital, which is defined as the ratio of the Lagrange multiplier of the evolution of capital to the marginal utility $\Lambda_{t}$.

Wage setting: The household supplies its differentiated labor service $\ell_{t}(h)$ to the intermediategood firms. Then, the labor market is monopolistically competitive. The intermediategood firm $f$ aggregates its labor inputs $\ell_{t}(f, h)$ according to the following technology:

$$
\ell_{t}(f)=\left[\int_{0}^{1} \ell_{t}(f, h)^{\frac{1}{1+\lambda_{t}^{w}}} d h\right]^{1+\lambda_{t}^{w}}
$$

where $\lambda_{t}^{w}$ is a time-varying parameter for the elasticity of substitution between labor supplies $\theta_{t}^{w}>1$, which is defined by $\lambda_{t}^{w}=1 /\left(\theta_{t}^{w}-1\right)>0$. The cost minimization of the intermediate-good firm and the aggregation over intermediate-good firms imply the following demand function of labor $\ell_{t}(h)$ :

$$
\ell_{t}(h)=\left[\frac{W_{t}(h)}{W_{t}}\right]^{-\frac{1+\lambda_{t}^{w}}{\lambda_{t}^{w}}} \ell_{t} .
$$


Calvo-type sticky wages are introduced as in Erceg, Henderson, and Levin (2000). In every period, a fraction $\xi_{w} \in[0,1]$ of households can reoptimize their wages. The other households index their wages to both the gross steady-state balanced growth rate $\left(z^{*}\right)$ and the weighted average of past inflation and the steady-state inflation : $\pi_{t-1}^{\gamma_{w}}\left(\pi^{*}\right)^{1-\gamma_{w}}$, where $\gamma_{w} \in[0,1]$ is the relative weight of the past inflation. The objective function is

$$
E_{t} \sum_{j=0}^{\infty}\left(\beta \xi_{w}\right)^{j}\left[\Lambda_{t+j} \ell_{t+j}(h)\left(\frac{P_{t} W_{t}(h)}{P_{t+j}}\right) \prod_{k=1}^{j}\left(z^{*} \pi_{t+k-1}^{\gamma_{w}}\left(\pi^{*}\right)^{1-\gamma_{w}}\right)-\frac{Z_{t+j}^{1-\sigma} \exp \left(z_{t+j}^{\ell}\right) \ell_{t+j}(h)^{1+\chi}}{1+\chi}\right],
$$

and the labor demand function is given by

$$
\ell_{t+j}(h)=\left[\frac{P_{t} W_{t}(h)}{P_{t+j} W_{t+j}} \prod_{k=1}^{j}\left(z^{*} \pi_{t+k-1}^{\gamma_{w}}\left(\pi^{*}\right)^{1-\gamma_{w}}\right)\right]^{-\frac{1+\lambda_{w}^{w}}{\lambda_{t}^{w}}} \ell_{t+j} .
$$

The reoptimized wage $W_{t}^{o}$ is the same for all households. The first-order condition for reoptimized wage $W_{t}^{o}$ is

$$
1=\frac{E_{t} \sum_{j=0}^{\infty}\left(\beta \xi_{w}\right)^{j} \frac{\left(1+\lambda_{t+j}^{w}\right) \exp \left(z_{t+j}^{b}\right) \exp \left(z_{t+j}^{e}\right) z_{t+j}^{1-\sigma}}{\lambda_{t+j}^{w}}\left[\ell_{t+j}\left[\frac{W_{t}^{0}\left(z^{*}\right)^{j}}{W_{t+j}} \prod_{k=1}^{j}\left(\frac{\pi_{t+k-1}}{\pi^{*}}\right)^{\gamma_{w}} \frac{\pi^{*}}{\pi_{t+k}}\right]^{-\frac{1+\lambda_{t+j}^{w}}{\lambda_{t+j}^{w}}}\right)^{1+\chi}}{E_{t} \sum_{j=0}^{\infty}\left(\beta \xi_{w}\right)^{j} \frac{\Lambda_{t+j} W_{t+j}}{\lambda_{t+j}^{w}} \ell_{t+j}\left[\frac{W_{t}^{o}\left(z^{*}\right)^{j}}{W_{t}} \prod_{k=1}^{j}\left(\frac{\pi_{t+k-1}}{\pi^{*}}\right)^{\gamma_{w}} \frac{\pi^{*}}{\pi_{t+k}}\right]^{-\frac{1}{\lambda_{t+j}^{w}}}} .
$$

Central bank: The central bank follows a Taylor-type nominal interest rate rule:

$$
\log R_{t}^{n}=\phi_{r} \log R_{t-1}^{n}+\left(1-\phi_{r}\right)\left[\log \bar{R}^{n}+\phi_{\pi} \frac{1}{4} \sum_{j=0}^{3} \log \frac{\pi_{t-j}}{\pi^{*}}+\phi_{y} \log \frac{Y_{t}}{Y_{t}^{P}}\right]+z_{t}^{r},
$$

where $\pi^{*}$ is the steady-state inflation rate; $Y_{t}^{P}$ is the potential output; and $z_{t}^{r}$ is the monetary policy shock. Parameter $\phi_{r} \in[0,1)$ represents the degree of interest rate smoothing, and $\phi_{\pi}>1$ and $\phi_{y} \geq 0$ are the monetary policy responses to inflation and output, respectively. The potential output $Y_{t}^{P}$ is defined by

$$
Y_{t}^{P}=\left[u^{*} k^{*} Z_{t-1}^{P}\right]^{\alpha}\left[Z_{t}^{P} \ell^{*}\right]^{1-\alpha}-\Phi Z_{t}^{P},
$$


where $u^{*}$ is the steady-state of capital utilization; $k^{*}$ is the steady-state detrended capital stock $\left(K_{t} / Z_{t}^{P}\right)$; and $\ell^{*}$ is the steady-state hours worked. This specification of the potential output is similar to the estimates of Hara et al. (2006). In this specification, only the permanent (technology) shock is considered as a driving force of potential output. This setup is similar to the estimates of Fueki et al. (2016).

Aggregations and market clearing conditions: Since the decisions on $u_{t}(h), I_{t}(h)$, and $K_{t}(h)$ are the same for all households, the evolution of the capital stock (12) is given by

$$
K_{t}=\left[1-\delta\left(u_{t}\right)\right] K_{t-1}+\left[1-S\left(\frac{I_{t}}{I_{t-1}} \frac{\exp \left(z_{t}^{i}\right)}{z^{*}}\right)\right] I_{t} .
$$

The capital market-clearing conditions are given by

$$
u_{t} K_{t-1}=\int_{0}^{1} K_{t}^{S}(f) d f .
$$

Combining the cost-minimization conditions of intermediate-good firms (5) and (6) and aggregation over intermediate-good firms yields

$$
\frac{1-\alpha}{\alpha}=\frac{W_{t} \ell_{t}}{R_{t}^{k} u_{t} K_{t-1}}
$$

The real marginal cost is given by

$$
m c_{t}=\frac{1}{\exp \left(z_{t}^{z t}\right)}\left(\frac{W_{t}}{(1-\alpha) Z_{t}^{P}}\right)^{1-\alpha}\left(\frac{R_{t}^{k}}{\alpha}\right)^{\alpha} .
$$

Aggregating the production function (3) over intermediate-good firms yields

$$
Y_{t} s_{t}=\exp \left(z_{t}^{z t}\right)\left[u_{t} K_{t-1}\right]^{\alpha}\left[Z_{t}^{P} \ell_{t}\right]^{1-\alpha}-\Phi Z_{t}^{P}
$$

where $s_{t}=\int_{0}^{1}\left[P_{t}(f) / P_{t}\right]^{-\left(1+\lambda_{t}^{p}\right) / \lambda_{t}^{p}} d f$ is the price dispersion of the intermediate-good price. This price dispersion can be ignored in the linearized system around the steady state where the steady-state value is one. 
Finally, the resource constraint is

$$
C_{t}+I_{t}+g^{*} Z_{t}^{P} \exp \left(z_{t}^{g}\right)=Y_{t}
$$

where $g^{*}$ is the steady-state ratio of government purchases to output; and $z_{t}^{g}$ is a government shock.

Labor wedge: Following Shimer (2009), the labor wedge is defined as follows.

$$
\text { (Labor wedge })=\frac{1}{1-\alpha}\left[\frac{C_{t}}{Y_{t}}\right] \ell_{t}^{\frac{1+\chi}{x}} .
$$

This specification is based on the period utility $\log \left(C_{t}(h)\right)-\frac{\ell_{t}(h)^{1+\chi}}{1+\chi}$, and the Cobb-Douglas production function.

\subsection{Log-Linearized Equilibrium Conditions}

The endogenous variables except for marginal utility $\Lambda_{t}$ are detrended by the technology level $Z_{t}$ as $x_{t}=X_{t} / Z_{t}$. The marginal utility $\Lambda_{t}$ is detrended as $\lambda_{t}=\Lambda_{t} / Z_{t}^{-\sigma}$. The equilibrium conditions are log-linearized around a steady state. The variable with tilde $\tilde{x}_{t}$ is defined as the log deviation of $x_{t}$ from its steady-state value $x^{*}$ :

$$
\tilde{x}_{t}=\log \left(x_{t}\right)-\log \left(x^{*}\right) .
$$

The log-linearized equilibrium system is described as follows. The marginal utility of consumption (14) is

$$
\begin{aligned}
\left(1-\frac{\theta}{z^{*}}\right)\left(1-\frac{\beta \theta}{\left(z^{*}\right)^{\sigma}}\right) \tilde{\lambda}_{t}= & -\sigma\left\{\tilde{c}_{t}-\frac{\theta}{z^{*}}\left(\tilde{c}_{t-1}-z_{t}^{z}\right)\right\}+\left(1-\frac{\theta}{z^{*}}\right) z_{t}^{b} \\
& +\frac{\beta \theta}{\left(z^{*}\right)^{\sigma}}\left[\sigma\left\{E_{t} \tilde{c}_{t+1}+E_{t} z_{t+1}^{z}-\frac{\theta}{z^{*}} \tilde{c}_{t}\right\}-\left(1-\frac{\theta}{z^{*}}\right) E_{t} z_{t+1}^{b}\right] .
\end{aligned}
$$

The Euler equation for nominal bond (15) is

$$
\tilde{\lambda}_{t}=E_{t} \tilde{\lambda}_{t+1}-\sigma E_{t} z_{t+1}^{z}+\tilde{R}_{t}^{n}-E_{t} \tilde{\pi}_{t+1}
$$


The capital utilization (16) is

$$
\tilde{u}_{t}=\mu\left(\tilde{R}_{t}^{k}-\tilde{q}_{t}\right)
$$

The Euler equation for investment (17) is

$$
\frac{1}{\zeta}\left\{\tilde{i}_{t}-\tilde{i}_{t-1}+z_{t}^{z}+z_{t}^{i}\right\}=\tilde{q}_{t}+\frac{\beta\left(z^{*}\right)^{1-\sigma}}{\zeta}\left\{E_{t} \tilde{i}_{t+1}-\tilde{i}_{t}+E_{t} z_{t+1}^{z}+E_{t} z_{t+1}^{i}\right\} .
$$

The Euler equation for capital (18) is

$$
\tilde{q}_{t}=E_{t} \tilde{\lambda}_{t+1}-\lambda_{t}-\sigma E_{t} z_{t+1}^{z}+\frac{\beta}{\left(z^{*}\right)^{\sigma}}\left\{R^{k} E_{t} \tilde{R}_{t+1}^{k}+(1-\delta) E_{t} \tilde{q}_{t+1}\right\} .
$$

The New Keynesian Phillips curve (9) is

$$
\tilde{\pi}_{t}-\gamma_{p} \tilde{\pi}_{t-1}=\beta\left(z^{*}\right)^{1-\sigma}\left(E_{t} \tilde{\pi}_{t+1}-\gamma_{p} \tilde{\pi}_{t}\right)+\frac{\left(1-\xi_{p}\right)\left(1-\beta \xi_{p} z^{1-\sigma}\right)}{\xi_{p}} \widetilde{m c}_{t}+z_{t}^{p} .
$$

where $z_{t}^{p}$ denotes the price markup shock, which is defined by

$$
z_{t}^{p}=\frac{\left(1-\xi_{p}\right)\left(1-\beta \xi_{p} \bar{z}^{1-\sigma}\right)}{\xi_{p}} \tilde{\lambda}_{t}^{p} .
$$

The New Keynesian wage Phillips curve (23) is

$$
\begin{aligned}
& \tilde{w}_{t}-\tilde{w}_{t-1}+\tilde{\pi}_{t}-\gamma_{w} \tilde{\pi}_{t-1}+z_{t}^{z} \\
& =\beta z^{1-\sigma}\left(E_{t} \tilde{w}_{t+1}-\tilde{w}_{t}+E_{t} \tilde{\pi}_{t+1}-\gamma_{w} \tilde{w}_{t}+E_{t} z_{t+1}^{z}\right) \\
& +\frac{1-\xi_{w}}{\xi_{w}} \frac{\left(1-\beta \xi_{w}\left(z^{*}\right)^{1-\sigma}\right) \lambda^{w}}{\lambda^{w}+\chi\left(1+\lambda^{w}\right)}\left(\chi \tilde{\ell}_{t}-\tilde{\lambda}_{t}-\tilde{w}_{t}+z_{t}^{b}\right)+z_{t}^{w},
\end{aligned}
$$

where $z_{t}^{w}$ denotes the wage shock, which is defined by

$$
z_{t}^{w}=\frac{1-\xi_{w}}{\xi_{w}} \times \frac{\left(1-\beta \xi_{w} \bar{z}^{1-\sigma}\right) \lambda^{w}}{\lambda^{w}+\xi\left(1+\lambda^{w}\right)}\left(\tilde{\lambda}_{t}^{w}+z_{t}^{\ell}\right)
$$

In the log-linearized equilibrium system, the wage markup shock $\lambda_{t}^{w}$ and the labor supply shocks $z_{t}^{\ell}$ cannot be identified. Then, the wage shock $z_{t}^{w}$ is defined as the linear combination of the (log-linearized) wage markup shock $\tilde{\lambda}_{t}^{w}$, where $\lambda^{w}$ is the steady-state 
value of $\lambda_{t}^{w}$ and labor supply shock $z_{t}^{\ell}$.

The monetary policy rule (24) is

$$
\tilde{R}_{t}^{n}=\phi_{r} \tilde{R}_{t-1}^{n}+\left(1-\phi_{r}\right)\left\{\phi_{\pi}\left(\frac{1}{4} \sum_{j=0}^{3} \tilde{\pi}_{t-j}\right)+\phi_{y}\left(\tilde{y}_{t}-\tilde{y}_{t}^{P}\right)\right\}+z_{t}^{r} .
$$

The potential output (25) is

$$
\tilde{y}_{t}^{P}=-\alpha(1+\phi) z_{t}^{z}
$$

where $\phi=\Phi / y^{*}$, and $y^{*}$ is the steady-state value of detrended output $Y_{t} / Z_{t}^{P}$. The evolution of aggregate capital (26) is

$$
\tilde{k}_{t}=\frac{1-\delta}{z^{*}}\left(\tilde{k}_{t-1}-z_{t}^{z}\right)-\frac{\bar{R}^{k}}{z^{*}} \tilde{u}_{t}+\left(1-\frac{1-\delta}{z^{*}}\right) \tilde{i}_{t} .
$$

The cost-minimization conditions (28) and (29) are

$$
\begin{aligned}
& \tilde{u}_{t}+\tilde{k}_{t-1}-\tilde{\ell}_{t}-z_{t}^{z p}=\tilde{w}_{t}-\tilde{R}_{t}^{k}, \\
& \widetilde{m c}_{t}=(1-\alpha) \tilde{w}_{t}+\alpha \tilde{R}_{t}^{k}-z_{t}^{z t} .
\end{aligned}
$$

The aggregate production function (30) is

$$
\tilde{y}_{t}=(1+\phi)\left\{z_{t}^{z t}+(1-\alpha) \tilde{\ell}_{t}+\alpha\left(\tilde{u}_{t}+\tilde{k}_{t-1}-z_{t}^{z}\right)\right\}
$$

The resource constraint (31) is

$$
\tilde{y}_{t}=\frac{c^{*}}{y^{*}} \tilde{c}_{t}+\frac{i^{*}}{y^{*}} \tilde{i}_{t}+\frac{g^{*}}{y^{*}} z_{t}^{g}
$$

There are seven (independent) exogenous shocks in the model. They follow the AR(1) 
process:

$$
\begin{aligned}
\text { permanent technology: } & z_{t}^{z p}=\rho_{z p} z_{t-1}^{z p}+\sigma_{z p} \varepsilon_{t}^{z p} \\
\text { transitory technology: } & z_{t}^{z t}=\rho_{z t} z_{t-1}^{z t}+\sigma_{z t} \varepsilon_{t}^{z t} \\
\text { preference: } & z_{t}^{b}=\rho_{b} z_{t-1}^{b}+\sigma_{b} \varepsilon_{t}^{b} \\
\text { government purchases: } & z_{t}^{g}=\rho_{g} z_{t-1}^{g}+\sigma_{g} \varepsilon_{t}^{g} \\
\text { investment adjustment cost: } & z_{t}^{i}=\rho_{i} z_{t-1}^{i}+\sigma_{i} \varepsilon_{t}^{i} \\
\text { price markup: } & z_{t}^{p}=\rho_{p} z_{t-1}^{p}+\sigma_{p} \varepsilon_{t}^{p} \\
\text { wage: } & z_{t}^{w}=\rho_{w} z_{t-1}^{w}+\sigma_{w} \varepsilon_{t}^{w} \\
\text { monetary policy: } & z_{t}^{r}=\rho_{r} z_{t-1}^{r}+\sigma_{r} \varepsilon_{t}^{r} .
\end{aligned}
$$

where $\sigma^{x} \varepsilon_{t}^{x}$ denotes a structural shock to $z_{t}^{x}$ for $x=z p, z t, b, g, i, p, w$, and $r$, and $\varepsilon_{t}^{x}$ is independently and identically distributed (i.i.d.), with a mean of zero and standard deviation of one.

\section{Data and Estimation Strategy}

We use seven quarterly Japanese series as observable variables: real GDP per capita $Y_{t}$, real consumption per capita $C_{t}$, real investment per capita $I_{t}$, real wage $W_{t}$, hours worked $\ell_{t}$, consumer price index $P_{t}$, and overnight call rate $R_{t}^{n}$. As in Hirose and Kurozumi (2012), the sample period is from 1981:Q1 to 1998:Q4. The model does not take into account the non-linearity of monetary policy. Then, the end of the sample period is set to exclude the zero nominal interest policy of the Bank of Japan.

Except for $I_{t}$ and $\ell_{t}$, the others series follow Hirose and Kurozumi (2012). $I_{t}$ is defined as per capita gross fixed capital formation by the private sector, which consists of private residential investment, private non-residential investment, and change in private inventories and is taken from the Economic and Social Research Institute, Cabinet Office of Japan, the System of National Accounts. $\ell_{t}$, following Hayashi and Prescott (2002) 
and Kobayashi and Inaba (2006), is constructed by

$$
\ell_{t}=\frac{\text { Averaged hours worked per employed person } \times \text { Employed person }}{\text { Labor force }} .
$$

The data source of average hours worked per employed person is the Ministry of Health, Labour and Welfare, Monthly Labor Survey. The employed persons and labor force data are from the Ministry of Internal Affairs and Communications, Labour Force Survey. Because our study focuses on the labor wedge, this definition of $\ell_{t}$ is commonly used for calculating the labor wedge; see, for example, Shimer (2009).

The observation equation is

$$
\left[\begin{array}{c}
100 \Delta \log Y_{t} \\
100 \Delta \log C_{t} \\
100 \Delta \log I_{t} \\
100 \Delta \log W_{t} \\
100 \log \ell_{t} \\
100 \Delta \log P_{t} \\
100 \Delta \log R_{t}^{n}
\end{array}\right]=\left[\begin{array}{c}
z^{*} \\
z^{*} \\
z^{*} \\
z^{*} \\
\ell^{*} \\
\pi^{*} \\
r^{*}+\pi^{*}
\end{array}\right]+\left[\begin{array}{c}
\tilde{y}_{t}-\tilde{y}_{t-1}+z_{t}^{z} \\
\tilde{c}_{t}-\tilde{c}_{t-1}+z_{t}^{z} \\
\tilde{i}_{t}-\tilde{i}_{t-1}+z_{t}^{z} \\
\tilde{w}_{t}-\tilde{w}_{t-1}+z_{t}^{z} \\
\tilde{\ell}_{t} \\
\tilde{\pi}_{t} \\
\tilde{R}_{t}^{n}
\end{array}\right]+\left[\begin{array}{c}
\sigma_{y}^{m e} m e_{t}^{y} \\
\sigma_{c}^{m e} m e_{t}^{c} \\
\sigma_{i}^{m e} m e_{t}^{i} \\
\sigma_{w}^{m e} m e_{t}^{w} \\
\sigma_{\ell}^{m e} m e_{t}^{\ell} \\
\sigma_{\pi}^{m e} m e_{t}^{\pi} \\
\sigma_{r n}^{m e} m e_{t}^{r n}
\end{array}\right]
$$

where $\sigma_{j}^{m e} m e_{t}^{j}$ denotes the measurement error of variable $j=y, c, i, w, \ell, \pi$, and $r n$. $m e_{t}^{j}$ is an i.i.d. shock in which the mean is zero and the standard deviation is one. The measurement errors are necessary to allow the non-existence of structural shocks in the estimation. Generally, the number of exogenous shocks must be equal to or greater than the number of observations. There are seven observations and eight (independent) structural shocks in this model. To allow the non-existence of two or more structural shocks, we need additional exogenous shocks: measurement errors to avoid stochastic singularity.

The growth rate of the labor wedge is calculated by the observations:

$$
100 \Delta \log \text { Labor Wedge }_{t}=100 \Delta \log C_{t}-100 \Delta \log Y_{t}+(1+\chi) \Delta \log \ell_{t} \text {. }
$$


Most of the model parameters are estimated, while the following aspects are fixed to avoid identification issues. The steady-state depreciation rate of capital stock is set to $\delta\left(u^{*}\right)=0.06 / 4$. The cost share of capital in the production function is set to $\alpha=0.37$. The steady-state wage markup is set to $\lambda^{w}=0.20$. These three parameter values are taken from Sugo and Ueda (2008). The steady-state ratio of government purchases to output is set to $g^{*}=0.30$, which is at the data mean.

Bayesian estimation is employed by using Dynare. The prior distributions of the parameters are in Tables 1 and 2. For the structural parameters $\sigma, \theta, \chi, 1 / \zeta, \mu, \Phi$, $\gamma_{w}, \xi_{p}, \gamma_{p}, \xi_{p}, \phi_{r}, \phi_{\pi}$, and $\phi_{y}$, the prior distributions are the same as those in Hirose and Kurozumi (2012). For the steady-state price markup $\lambda^{p}$, the mean and standard deviation of the prior distribution are taken from Justiniano, Primiceri, and Tambalotti (2011), but the gamma distribution is employed to fit the theory. For the steady-state growth rate $z^{*}$, labor supply $\ell^{*}$, inflation $\pi^{*}$, and real interest $r^{*}$, the means of prior distribution are set at the sample mean. For the persistence parameters of structural shocks, the prior distribution is the beta distribution with a mean of 0.5 and a standard deviation of 0.2. For the parameters of standard deviations of structural shocks $\sigma_{x}$ and those for measurement errors $\sigma_{j}^{m e}$, we consider two types of prior distributions. One is the inverse gamma distribution, which is standard in the literature, with a mean of 0.5 and a standard deviation of infinity. The other is normal distribution. For the parameter of standard deviations of structural shocks $\sigma_{x}$, the mean is 0.1 and the standard deviation is 10 . For the parameter of standard deviations of measurement errors $\sigma_{j}^{m e}$, the mean is $\sigma_{j}^{\text {data }} / 10$ and the standard deviation is $\sigma_{j}^{\text {data }}$, where $\sigma_{j}^{\text {data }}$ is the standard deviation of observations. Because the inverse gamma distribution does not support zero values, it assumes the existence of structural shocks and measurement errors. The normal distribution supports zero value, and then, allows the non-existence of structural shocks. Measurement errors are considered only in the case of normal distribution.

[Tables 1 and 2] 
Following standard Bayesian likelihood approaches, the Kalman filter is used to evaluate the likelihood function of the log-linearized equilibrium system and the MetropolisHastings algorithm is employed to generate draws from the posterior distribution of the deep parameters. For the ensuing analysis, 1 million draws are generated and the first half of them are discarded. The target of the acceptance rate is about $30 \%$.

\section{Empirical Results}

The posterior estimates are presented in Tables 3 and 4. Distribution (1) is the case in which inverse gamma distribution is employed as the prior distribution of the parameters for the standard deviation of structural shocks $\sigma_{x}$. Distribution (2) is the case in which normal distribution is employed as the prior distribution of the parameters for the standard deviations of structural shocks $\sigma_{x}$ and measurement errors $\sigma_{j}^{m e}$.

[Tables 3 and 4]

In Table 3, most estimates are similar in the two prior distributions. Table 4 implies that, in the case of Distribution (2), some structural shocks (transitory technology, preference, government spending, price markup, and wage shocks) might not exist in the sense in which the zero value lies inside the $90 \%$ credible intervals of the parameters for the standard deviations. The posterior mean is also close to zero for these parameters except for $\sigma_{b}$ (preference shock).

Our result is similar to that of Ferroni, Grassi, and Leon-Ledesma (2019), who find that the posterior means of the parameters for the standard deviations of the government spending, price markup, and wage markup shocks are close to zero in the US economy using Smets and Wouters' (2007) model. Our result is also related to that of Justiniano, Primiceri, and Tambalotti (2013), who find that the variation of the wage markup shock becomes minor if measurement errors are included in the observation equations. ${ }^{4}$

\footnotetext{
${ }^{4}$ Justiniano, Primiceri, and Tambalotti (2013) employ inverse gamma distribution as a prior distribution
} 
As discussed by Justiniano, Primiceri, and Tambalotti (2013), there is no trade-off between inflation and output stabilization if there is no wage markup shock. Chari, Kehoe, and McGrattan (2009) doubt the existence of a wage markup shock as a fundamental structural shock. Our estimate implies that the wage shock (including wage markup shock) might not exist as their conjecture. However, the labor wedge is driven by other structural shocks in our model.

Table 5 shows the variance decomposition of the output growth $\left(100 \Delta \log Y_{t}\right)$ and the labor wedge (100 $\Delta \log$ labor wedge). As in Tables 3 and 4, Distribution (1) shows the case in which inverse gamma distribution is employed as the prior distribution of standard deviation of structural shocks $\sigma_{x}$, and Distribution (2) is the case in which normal distribution is employed as the prior distribution of standard deviation of structural shocks $\sigma_{x}$ and measurement errors $\sigma_{j}^{m e}$.

\section{[Table 5]}

In the case of Distribution (1), the main driving force of output fluctuation is the investment adjustment cost shock. The preference, government, and permanent technology shocks are also important. The importance of the investment adjustment cost shock is consistent with Justiniano, Primiceri, and Tambalotti (2010, 2011) and Hirose and Kurozumi (2012). However, the main driving force of the labor wedge is the preference and transitory technology shocks. Then, the driving force of the labor wedge is different from that of output fluctuation. According to this result, the investigation of the labor wedge is not promising to understand the business cycle fluctuations.

In the case of Distribution (2), the main driving force of both output fluctuation and the labor wedge is the permanent technology shock. This finding is consistent with Kaihatsu and Kurozumi (2014a, 2014b), who find that the technology shock is the main driving force of business cycles both in the US and Japan. The investment adjustment of the parameters for the standard deviations of shocks, while this study employs normal distribution to allow the non-existence of shocks. 
cost shock is also important for these two variables, as in the result of Distribution (1). According to this estimate, output and the labor wedge are mainly driven by the same structural shocks. In this study, Distribution (2) is considered as a better prior distribution, since it allows the non-existence of structural shocks. Therefore, the investigation of the labor wedge fluctuations is promising to understand the business cycles.

Table 6 shows the marginal log likelihood in two estimations: Distributions (1) and (2). According to this table, our prior distribution, the normal distribution, is better than the inverse gamma distribution, which is standard in the literature, as shown by Ferroni, Grassi, and Leon-Ledesma (2019).

\section{Concluding remarks}

The labor wedge has been investigated by many researchers as an important variable to understand business cycle fluctuations. In this study, we estimated the main sources of the labor wedge and business cycle in the Japanese economy using a canonical mediumscale DSGE model with many nominal and real frictions and many structural shocks. We employed a more flexible prior distribution of the parameters for the standard deviations of structural shocks to allow the non-existence of structural shocks. However, in standard Bayesian estimation, the standard prior distribution of the parameters for the standard deviations of the shocks is inverse gamma distribution, which does not support zero value.

Under the standard prior distribution of the parameters for the standard deviations of structural shocks, our estimation results imply that the labor wedge is mainly driven by preference and transitory technology shocks, whereas the business cycle is mainly driven by the investment adjustment cost shock. Meanwhile, under our relaxed prior distribution, which allows the non-existence of structural shocks, our estimation results show that both the labor wedge and business cycles are mainly driven by the permanent 
technology and investment adjustment cost shocks. Our results also imply that the investigation of the labor wedge is promising to understand the business cycles, because both the labor wedge and business cycles are driven by the same structural shocks.

\section{References}

[1] Chari, V.V., Patrick J. Kehoe, and Ellen R. McGrattan. 2002. "Accounting for the Great Depression.” American Economic Review 92, 22-27.

[2] Chari, V.V., Patrick J. Kehoe, and Ellen R. McGrattan. 2007. "Business Cycle Accounting." Econometrica 75(3), 781-836.

[3] Chari, V.V., Patrick J. Kehoe, and Ellen R. McGrattan. 2009. "New Keynesian Models: Not Yet Useful for Policy Analysis.” American Economic Journal: Macroeconomics 1(1), 242-266.

[4] Christiano, Lawrence, J, Martin Eichenbaum, and Charles L. Evans. 2005. "Nominal Rigidities and the Dynamic Effects of a Shock to Monetary Policy.” Journal of Political Economy 113(1), 1-4.

[5] Cheremukhin, Anton A., and Paulina Restrepo-Echavarria. 2014. "The Labor Wedge as a Matching Friction.” European Economic Review 68, 71-92.

[6] Duras, Jun. 2017. "Goods Market Frictions and the Labor Wedge.” mimeo.

[7] Erceg, Christopher, J., Dale W. Henderson, and Andrew T. Levin. 2000. “Optimal Monetary Policy with Staggered Wage and Price Contracts.” Journal of Monetary Economics 46, 281-313.

[8] Erceg, Christopher, J., Luca Guerrieri, and Christopher Gust. 2006. "SIGMA: A New Open Economy Model for Policy Analysis.” International Journal of Central Banking 113, 1-45. 
[9] Ferroni, Filippo, Stefano Grassi, and Miguel Leon-Ledesma. 2019. "Selecting Structural Innovations in DSGE models.” Journal of Applied Econometrics 34(2), 205-220.

[10] Fueki, Takuji, Ichiro Fukunaga, Hibiki Ichiue, and Toyoichiro Shirota. 2016. "Measuring Potential Growth with an Estimated DSGE Model of Japan's Economy" International Journal of Central Banking 12(1), 1-32.

[11] Greenwood, Jeremy, Zvi Hercowitz, and Gregory W. Huffman. 1988. "Investment, Capacity Utilization, and the Real Business Cycle.” American Economic Review $78,402-417$.

[12] Hall, Robert, E. 2009. "Reconciling Cyclical Movements in the Marginal Value of Time and the Marginal Product of Labor." Journal of Political Economy 117(2), $282-323$.

[13] Hara, Naoko., Naohisa Hirakata, Yusuke Inomata, Satoshi Ito, Takuji Kawamoto, Takushi Kurozumi, Makoto Minegishi, and Izumi Takagawa. 2006. “The New Estimates of Output Gap and Potential Growth Rate.” Bank of Japan Review 2006E-3.

[14] Hayashi, Fumio and Edward C. Prescott. 2002. "The 1990s in Japan: A Lost Decade.” Review of Economic Dynamics 5(1), $206 ? 235$.

[15] Hirose, Yasuo and Takushi Kurozumi. 2012. "Do Investment-Specific Technological Changes Matter for Business Cycle Fluctuations?: Evidence from Japan.” Pacific Economic Review 17, 203-220.

[16] Justiniano, Alejandro, Giorgio E. Primiceri, and Andrea Tambalotti, 2010. "Investment Shocks and Business Cycles." Journal of Monetary Economics, 57, 132-145. 
[17] Justiniano, Alejandro, Giorgio E. Primiceri, and Andrea Tambalotti, 2011. "Investment Shocks and the Relative Price of Investment." Review of Economic Dynamics $14,102-121$.

[18] Justiniano, Alejandro, Giorgio E. Primiceri, and Andrea Tambalotti, 2013. "Is There a Trade-Off between Inflation and Output Stabilization?." American Economic Journal: Macroeconomics, 5(2), 1-31.

[19] Kaihatsu, Sohei, and Takushi Kurozumi. 2014a. "Sources of business fluctuations: Financial or technology shocks?” Review of Economic Dynamics 17, 224-242.

[20] Kaihatsu, Sohei, and Takushi Kurozumi. 2014b. "What caused Japan's Great Stagnation in the 1990s? Evidence from an estimated DSGE model." Journal of the Japanese and International Economies 34, 217-235.

[21] Karabarbounis, Loukas. 2014a. "The Labor Wedge: MRS vs. MPN.” Review of Economic Dynamics 17, 206-223.

[22] Karabarbounis, Loukas. 2014b. "Home Production, Labor Wedges, and International Business Cycles.” Journal of Monetary Economics 64, 68-84.

[23] King, Robert G., and Sergio T. Rebelo. 1999. "Resuscitating Real Business Cycles.” In: Taylor, J.B., Woodford, M. (Eds.), Handbook of Macroeconomics. Elsevier, Amsterdam, 927-1007.

[24] Kobayashi, Keiichiro, and Masaru Inaba. 2006. "Business Cycle Accounting for the Japanese Economy.” Japan and the World Economy 18, 418-440.

[25] Ohanian, Lee E., 2010. "The Economic Crisis from a Neoclassical Perspective." Journal of Economic Perspectives 24 (4), 45-66.

[26] Otsu, Keisuke. 2011. "Accounting for Japanese business cycles; a quest for labor wedges." Monetary and Economic Studies 29, 143-170. 
[27] Pescatori, Andrea, and Murat Tasci. 2011. "Search Frictions and the Labor Wedge.” IMF Working Paper 11/117.

[28] Shimer, Robert. 2009. "Convergence in Macroeconomics: The Labor Wedge." American Economic Journal: Macroeconomics 1:1 280-287.

[29] Smets, Frank, and Rafael Wouters. 2007. "Shocks and Frictions in U.S. Business Cycles: A Bayesian DSGE Approach.” American Economic Review 97, 586-606.

[30] Sugo, Tomohiro, and Kozo Ueda, 2008. "Estimating a Dynamic Stochastic General Equilibrium Model for Japan.” Journal of the Japanese and International Economies 22, 476-502.

[31] Zhang, Lini. 2018. "Credit Crunches, Individual Heterogeneity and the Labor Wedge.” Journal of Macroeconomics 56, 65-88. 
Table 1: Prior Distribution (1/2)

\begin{tabular}{|c|c|c|c|c|}
\hline & Parameter & Distribution & Mean & $\mathrm{SD}$ \\
\hline$\sigma$ & Relative risk aversion & Gamma & 1.0000 & 0.3750 \\
\hline$\theta$ & Habit persistence & Beta & 0.7000 & 0.1500 \\
\hline$\chi$ & Inverse of Frisch elasticity of labor supply & Gamma & 2.0000 & 0.7500 \\
\hline $1 / \zeta$ & Inverse of adj. cost of investment & Gamma & 4.0000 & 1.5000 \\
\hline$\mu$ & Inverse of elasticity of utilization adj. cost & Gamma & 1.0000 & 1.0000 \\
\hline$\Phi$ & Fixed cost in production function & Gamma & 0.0750 & 0.0125 \\
\hline$\gamma_{w}$ & Wage indexation & Beta & 0.5000 & 0.2500 \\
\hline$\xi_{w}$ & Wage stickiness & Beta & 0.3750 & 0.1000 \\
\hline$\gamma_{p}$ & Price indexation & Beta & 0.5000 & 0.2500 \\
\hline$\xi_{p}$ & Price stickiness & Beta & 0.3750 & 0.1000 \\
\hline$\lambda^{p}$ & Steady-state price markup & Gamma & 0.1500 & 0.0500 \\
\hline$z^{*}$ & Steady-state output growth & Gamma & 0.353 & 0.0500 \\
\hline$\ell^{*}$ & Steady-state hours worked & Normal & 0.0000 & 0.0500 \\
\hline$\pi^{*}$ & Steady-state inflation & Gamma & 0.341 & 0.0500 \\
\hline$r^{*}$ & Steady-state real interest rate & Gamma & 1.088 & 0.0500 \\
\hline$\phi_{r}$ & Interest rate smoothing & Beta & 0.8000 & 0.1000 \\
\hline$\phi_{\pi}$ & Monetary policy response to inflation & Gamma & 1.7000 & 0.1000 \\
\hline$\phi_{y}$ & Monetary policy response to output & Gamma & 0.125 & 0.0500 \\
\hline
\end{tabular}


Table 2: Prior Distribution (2/2)

\begin{tabular}{|c|c|c|c|c|c|c|c|}
\hline \multirow{2}{*}{\multicolumn{2}{|c|}{ Parameter }} & \multicolumn{3}{|c|}{ Distribution (1) } & \multicolumn{3}{|c|}{ Distribution (2) } \\
\hline & & & Mean & $\mathrm{SD}$ & & Mean & SD \\
\hline$\rho_{z p}$ & Persistence of permanent technology shock & Beta & 0.5 & 0.2 & Beta & 0.5 & 0.2 \\
\hline$\rho_{z t}$ & Persistence of transitory technology shock & Beta & 0.5 & 0.2 & Beta & 0.5 & 0.2 \\
\hline$\rho_{b}$ & Persistence of preference shock & Beta & 0.5 & 0.2 & Beta & 0.5 & 0.2 \\
\hline$\rho_{i}$ & Persistence of investment adj. cost shock & Beta & 0.5 & 0.2 & Beta & 0.5 & 0.2 \\
\hline$\rho_{g}$ & Persistence of government shock & Beta & 0.5 & 0.2 & Beta & 0.5 & 0.2 \\
\hline$\rho_{w}$ & Persistence of wage shock & Beta & 0.5 & 0.2 & Beta & 0.5 & 0.2 \\
\hline$\rho_{p}$ & Persistence of price markup shock & Beta & 0.5 & 0.2 & Beta & 0.5 & 0.2 \\
\hline$\rho_{r}$ & Persistence of monetary policy shock & Beta & 0.5 & 0.2 & Beta & 0.5 & 0.2 \\
\hline$\sigma_{z p}$ & SD of permanent technology shock & IG & 0.5 & $\operatorname{Inf}$ & Normal & 0.1 & 10 \\
\hline$\sigma_{z t}$ & SD of transitory technology shock & IG & 0.5 & $\operatorname{Inf}$ & Normal & 0.1 & 10 \\
\hline$\sigma_{b}$ & SD of preference shock & IG & 0.5 & Inf & Normal & 0.1 & 10 \\
\hline$\sigma_{i}$ & SD of investment adj. cost shock & IG & 0.5 & Inf & Normal & 0.1 & 10 \\
\hline$\sigma_{g}$ & SD of government shock & IG & 0.5 & $\operatorname{Inf}$ & Normal & 0.1 & 10 \\
\hline$\sigma_{w}$ & SD of wage shock & IG & 0.5 & Inf & Normal & 0.1 & 10 \\
\hline$\sigma_{p}$ & SD of price markup shock & IG & 0.5 & Inf & Normal & 0.1 & 10 \\
\hline$\sigma_{r}$ & SD of monetary policy shock & IG & 0.5 & Inf & Normal & 0.1 & 10 \\
\hline$\sigma_{y}^{m e}$ & SD of ME of GDP & NA & NA & NA & Normal & $\sigma_{y}^{d a t a} / 10$ & $\sigma_{y}^{d a t a}$ \\
\hline$\sigma_{c}^{m e}$ & SD of ME of consumption & NA & NA & NA & Normal & $\sigma_{c}^{d a t a} / 10$ & $\sigma_{c}^{d a t a}$ \\
\hline$\sigma_{i}^{m e}$ & SD of ME of investment & NA & NA & NA & Normal & $\sigma_{i}^{\text {data }} / 10$ & $\sigma_{i}^{\text {data }}$ \\
\hline$\sigma_{w}^{m e}$ & SD of ME of wage & NA & NA & NA & Normal & $\sigma_{w}^{d a t a} / 10$ & $\sigma_{w}^{d a t a}$ \\
\hline$\sigma_{\ell}^{m e}$ & SD of ME of hours worked & NA & NA & NA & Normal & $\sigma_{\ell}^{\text {data }} / 10$ & $\sigma_{\ell}^{\text {data }}$ \\
\hline$\sigma_{\pi}^{m e}$ & SD of ME of inflation & NA & NA & NA & Normal & $\sigma_{\pi}^{\text {data }} / 10$ & $\sigma_{\pi}^{\text {data }}$ \\
\hline$\sigma_{r n}^{m e}$ & SD of ME of call rate & NA & NA & $\mathrm{NA}$ & Normal & $\sigma_{r n}^{\text {data }} / 10$ & $\sigma_{r n}^{\text {data }}$ \\
\hline
\end{tabular}

Notes: IG denotes the Inverse Gamma distribution. ME denotes measurement error. $\sigma_{x}^{\text {data }}$ means the standard deviation of actual data for $x=y, c, i, w, \ell, \pi$, and $r n$. 
Table 3: Posterior Estimates (1/2)

\begin{tabular}{|c|c|c|c|c|}
\hline & \multicolumn{2}{|c|}{$\begin{array}{c}\text { Distribution (1) } \\
\text { no Measurement Errors } \\
\text { (Prior on } \sigma_{x}: \text { Inverse Gamma) }\end{array}$} & \multicolumn{2}{|c|}{$\begin{array}{c}\text { Distribution (2) } \\
\text { with Measurement Errors } \\
\text { (Prior on } \sigma_{x} \text { and } \sigma_{j}^{m e}: \text { Normal) }\end{array}$} \\
\hline & Mean & $90 \%$ credible interval & Mean & $90 \%$ credible interval \\
\hline$\sigma$ & 1.8176 & {$[0.2985,3.3961]$} & 2.3811 & [ $1.6397,3.1031]$ \\
\hline$\theta$ & 0.6795 & {$[0.2730,0.9815]$} & 0.3660 & {$[0.1837,0.5416]$} \\
\hline$\chi$ & 2.1229 & {$[1.2790,3.0523]$} & 1.3495 & {$[0.6600,2.0457]$} \\
\hline $1 / \zeta$ & 6.8186 & {$[3.3844,10.0621]$} & 4.1703 & {$[1.8334,6.3872]$} \\
\hline$\mu$ & 2.1878 & {$[0.4406,3.8635]$} & 0.0705 & {$[0.0000,0.1595]$} \\
\hline$\Phi$ & 0.0776 & {$[0.0559,0.0969]$} & 0.0739 & {$[0.0543,0.0936]$} \\
\hline$\gamma_{w}$ & 0.5891 & {$[0.2273,0.9791]$} & 0.4347 & {$[0.0248,0.8217]$} \\
\hline$\xi_{w}$ & 0.5965 & {$[0.5131,0.6786]$} & 0.4664 & {$[0.3246,0.6080]$} \\
\hline$\gamma_{p}$ & 0.4481 & {$[0.1126,0.7982]$} & 0.4034 & {$[0.0174,0.7739]$} \\
\hline$\xi_{p}$ & 0.705 & {$[0.6407,0.7655]$} & 0.5307 & {$[0.3590,0.7103]$} \\
\hline$\lambda^{p}$ & 0.3066 & {$[0.1882,0.4234]$} & 0.1494 & {$[0.0687,0.2252]$} \\
\hline$z^{*}$ & 0.3222 & {$[0.2530,0.3900]$} & 0.4777 & {$[0.3907,0.5701]$} \\
\hline$\ell^{*}$ & -0.0005 & {$[-0.0828,0.0820]$} & -0.0022 & {$[-0.0857,0.0803]$} \\
\hline$\pi^{*}$ & 0.3586 & {$[0.2725,0.4461]$} & 0.3427 & {$[0.2588,0.4236]$} \\
\hline$r^{*}$ & 1.0655 & {$[0.9863,1.1400]$} & 1.0119 & {$[0.9367,1.0871]$} \\
\hline$\phi_{r}$ & 0.6897 & {$[0.5943,0.7849]$} & 0.6090 & {$[0.5058,0.7182]$} \\
\hline$\phi_{\pi}$ & 1.7645 & {$[1.6089,1.9194]$} & 1.7154 & {$[1.5632,1.8679]$} \\
\hline$\phi_{y}$ & 0.1064 & {$[0.0426,0.1932]$} & 0.1163 & {$[0.0616,0.1697]$} \\
\hline
\end{tabular}


Table 4: Posterior Estimates (2/2)

\begin{tabular}{|c|c|c|c|c|}
\hline & \multicolumn{2}{|c|}{$\begin{array}{c}\text { Distribution (1) } \\
\text { no Measurement Errors } \\
\text { (Prior on } \sigma_{x} \text { : Inverse Gamma) }\end{array}$} & \multicolumn{2}{|c|}{$\begin{array}{c}\text { Distribution (2) } \\
\text { with Measurement Errors } \\
\text { (Prior on } \sigma_{x} \text { and } \sigma_{j}^{m e} \text { : Normal) }\end{array}$} \\
\hline & Mean & $90 \%$ credible interval & Mean & $90 \%$ credible interval \\
\hline$\rho_{z p}$ & 0.5532 & {$[0.2259,0.8721]$} & 03854 & {$[0.1779,0.5799]$} \\
\hline$\rho_{z t}$ & 0.9613 & {$[0.9361,0.9864]$} & 0.4739 & {$[0.1031,0.8281]$} \\
\hline$\rho_{b}$ & 0.3091 & {$[0.0191,0.6505]$} & 0.6274 & {$[0.2774,0.9948]$} \\
\hline$\rho_{i}$ & 0.5005 & {$[0.2382,0.7497]$} & 0.8813 & {$[0.8147,0.9506]$} \\
\hline$\rho_{g}$ & 0.7292 & {$[0.3163,0.9946]$} & 0.4719 & {$[0.0822,0.8315]$} \\
\hline$\rho_{w}$ & 0.1049 & {$[0.0138,0.1976]$} & 0.7633 & {$[0.3516,0.9892]$} \\
\hline$\rho_{p}$ & 0.9462 & {$[0.9078,0.9853]$} & 0.4963 & {$[0.1661,0.8395]$} \\
\hline$\rho_{r}$ & 0.539 & {$[0.3479,0.7348]$} & 0.4162 & {$[0.2233,0.6027]$} \\
\hline$\sigma_{z p}$ & 0.5216 & {$[0.1752,0.8636]$} & 1108 & {$[0.8277,1.5184]$} \\
\hline$\sigma_{z t}$ & 0.8466 & {$[0.7051,0.9$} & 0056 & $, 0.2548]$ \\
\hline$\sigma_{b}$ & 9.3614 & {$[0.1110,20.7598]$} & -0.1434 & {$[-1.8223,1.6020]$} \\
\hline$\sigma_{i}$ & 5.3242 & {$[3.6466,7.0190]$} & 7.1110 & {$[2.9951,11.2634]$} \\
\hline$\sigma_{g}$ & 1.5095 & {$[1.0323,1.9465]$} & 0.0388 & {$[-0.9004,0.9858]$} \\
\hline$\sigma_{w}$ & 0.9992 & {$[0.8245,1.1594]$} & 0.0369 & {$[-0.2661,0.3446]$} \\
\hline$\sigma_{p}$ & 0.1569 & {$[0.0937,0.2253]$} & -0.0025 & {$[-0.2026,0.1987]$} \\
\hline$\sigma_{r}$ & 0.1192 & {$[0.1006,0.1368]$} & 0.0939 & {$[0.0714,0.1169]$} \\
\hline$\sigma_{y}^{m e}$ & NA & NA & 0.1213 & {$[-0.5871,0.6286]$} \\
\hline$\sigma_{c}^{m e}$ & NA & NA & 0.0463 & {$[-0.6649,0.6937]$} \\
\hline$\sigma_{i}^{m e}$ & NA & NA & 2.7165 & {$[2.2638,3.1748]$} \\
\hline$\sigma_{g}^{m e}$ & NA & NA & 0.8827 & {$[0.7491,1.0164]$} \\
\hline$\sigma_{\ell}^{m e}$ & NA & NA & -0.0972 & {$[-0.4815,0.4505]$} \\
\hline$\sigma_{\pi}^{m e}$ & NA & NA & 0.0109 & {$[-0.1614,0.1673]$} \\
\hline$\sigma_{r n}^{m e}$ & NA & NA & 0.0007 & {$[-0.0459,0.0463]$} \\
\hline
\end{tabular}


Table 5: Variance Decompositions

\begin{tabular}{|c|c|c|c|c|}
\hline & \multicolumn{2}{|c|}{$\begin{array}{c}\text { Distribution (1) } \\
\text { no Measurement Errors } \\
\text { (Prior on } \sigma^{x} \text { : Inverse Gamma) }\end{array}$} & \multicolumn{2}{|c|}{$\begin{array}{c}\text { Distribution (2) } \\
\text { with Measurement Errors } \\
\text { (Prior on } \sigma^{x} \text { and } \sigma_{m e}^{j} \text { : Normal) }\end{array}$} \\
\hline & $100 \Delta \log Y_{t}$ & $100 \Delta \log$ Labor Wedge $_{t}$ & $100 \Delta \log Y_{t}$ & $100 \Delta \log$ Labor Wedge $_{t}$ \\
\hline$z^{b}$ & 28.02 & 50.50 & 0.02 & 0.38 \\
\hline$z^{i}$ & 34.93 & 5.74 & 12.55 & 19.59 \\
\hline$z^{g}$ & 9.68 & 4.70 & 0.01 & 0.08 \\
\hline$z^{w}$ & 0.76 & 7.71 & 0.08 & 0.95 \\
\hline$z^{p}$ & 4.99 & 5.31 & 0.00 & 0.00 \\
\hline$z^{r}$ & 0.21 & 2.97 & 0.24 & 4.53 \\
\hline$z^{z p}$ & 12.92 & 0.86 & 85.80 & 62.40 \\
\hline$z^{z t}$ & 8.49 & 22.20 & 0.00 & 0.05 \\
\hline
\end{tabular}

Table 6: Marginal Log Likelihoods in Two Estimations

\begin{tabular}{cc}
\hline \hline $\begin{array}{c}\text { Distribution (1) } \\
\text { no Measurement Errors }\end{array}$ & Distribution (2) \\
(Prior on $\sigma_{x}:$ Inverse Gamma) & with Measurement Errors \\
\hline-570.393 & -549.151 \\
\hline \hline
\end{tabular}

